\title{
Color Recognition for NAO Robot Using Sugeno Fuzzy System and Evidence Theory
}

\author{
T.L. NGUYEN R. BOUKEZZOULA D. COQUIN S. PERRIN \\ LISTIC laboratory, Polytech, University of Savoie Mont Blanc, 74940 Annecy-le-vieux, France. \\ \{thanh-long.nguyen, reda.boukezzoula, didier.coquin, stephane.perrin\}@univ-savoie.fr
}

\begin{abstract}
This paper examines the problem of recognition of colored objects for the NAO humanoid robot using cameras. Based on the ability of fuzzy systems to approximate any mapping, the input-output behavior between the triplet $\{H, S, V\}$ and the linguistic output colors is represented by a Sugeno fuzzy system. In order to improve the recognition performance of the robot, more cameras are used. In this case, a decision mechanism based on the Dempster-Shafer (DS) theory is employed to resolve the conflict between cameras. The feasibility of the proposed methodology has been experimentally validated with a real-time implementation.
\end{abstract}

Keywords: NAO robot, Sugeno fuzzy system, Dempster-Shafer (DS), color recognition, information fusion.

\section{Introduction}

Nowadays, intelligent and humanoid mobile robots are used in numerous engineering domains such as industrial applications, service robots in home and office environments. However, developing a robot with good behavior following human perception is not an easy task and it represents a challenge to be solved. Indeed, the available data from the robot sensors integrate significant amounts of imprecision and/or uncertainty due to the environment, the changing in operating conditions, the characteristics of the sensors and the techniques employed to extract the data from them. In this paper, we consider developing an application that helps a NAO robot equipped with a camera to recognize the color of a required object. In this context, it seems that Artificial Intelligent techniques (AI) are plausible and reasonable tools to solve this problem [9][10][11][14]. That is the approach proposed in this paper where a humanoid NAO robot platform from the Aldebaran Robotics company is used to illustrate the performance and ability of the developed methodology.

Color recognition for robots is not a new problem. Indeed, this has been tackled in several researches and applications, like soccer or mobile robots in [8][17][19]. In these works, simple recognition techniques like color thresholding or region segmentation have been used. There is no doubt that these methods are simple and convenient but they take into account a lot of fixed parameters obtained through several experimentations. For most practical applications, it is a substantial task which is time consuming. Moreover, human reasoning and perception which are fundamental in humanoid mobile robots are not approached properly.

Since the color recognition is executed under uncertain and imprecise environments where an exact analytical procedure design cannot be determined, artificial intelligent (AI) methods like fuzzy systems and/or neural networks [10][12] seem to be very effective tools to develop color recognition strategies. Recently, such intelligent techniques have been successfully used for color recognition in RGB, HSV and Lab spaces. For instance in [9][10][11], Mamdani fuzzy systems are used for solving the color recognition problem. The basic idea of these works is to construct membership functions to determine the degree that a triplet in RGB, HSV or Lab spaces belongs to corresponding humandefined labels. Then a Mamdani inference mechanism is used to assess the detected output colors. It is important to note that most applications in the color recognition problem using fuzzy systems are based on the fuzzy Mamdani type where the nonlinear min/max operators are used in the inference mechanism. In the context of our research on color recognition, we did not find any advantage to exploit the $\mathrm{min} / \mathrm{max}$ operators. Moreover, the use of the latter operators involves the appearance of some nonlinearity which may potentially deteriorate the performances of our application.

Taking advantage of AI approaches, the work proposed in this paper is inspired from those quoted previously. Indeed, a Sugeno fuzzy system [1][16] in HSV space is used for recognizing the color of a target object by the NAO robot camera. The choice of HSV comes from that fact it is more intuitive to human eyes than basic color spaces like RGB. In HSV, Hue $(\mathrm{H})$ represents the color tone, Saturation $(\mathrm{S})$ is the amount of color and Value (V) denotes the lightness.

The choice of the Sugeno system comes from the need for good calculation performance. Moreover, the Sugeno fuzzy system presents structural properties [7] that allow exact piecewise multi-linear representation. This representation permits us to integrate this fuzzy system design in some adaptive or learning strategies to specify the rule base parameters. For example, adaptive and learning fuzzy strategy can be used in the future to adjust some parameters so that the recognition system performance is improved.

It is obvious that intelligent techniques can provide good results because they take into account human reasoning in color recognition behavior. However, with only one information source, they may also introduce problems of reliability and performance in presence of uncertainties and/or imprecisions (imprecise observations and uncertain measurements due to the operating 
conditions, the hesitation between neighboring colors, the camera's orientation ... etc.). These difficulties weaken the potential use of AI methods in real applications. For example recognizing colors under different lighting conditions may affect the quality and the reliability of recognition.

Since a single source of information (one camera) is not enough to reflect the real color (almost all of the previously described papers do not consider this problem), in this context additional sensors must be employed to acquire more exact information. Therefore, we propose to add a displaced IP camera as the second information source. After that, Evidence Theory (Dempster-Shafer theory (DS)) [4][5][6][18] is employed to fuse information between the two cameras in order to solve the conflicts and uncertainties. The DS theory is a very useful method to express and fuse the uncertainty information, and is especially suitable for decision level fusion.

The paper is organized as follow. Section 2 presents Sugeno fuzzy system for color recognition using a camera in HSV space. Section 3 is devoted to the application of Evidence Theory to overcome the conflict problem between cameras. After that, section 4 shows the experimental results. Section 5 gives the conclusion and some perspectives.

\section{Sugeno Fuzzy System for Color Recognition}

\subsection{Fuzzy system architecture}

In the proposed methodology, a Sugeno fuzzy system with constant conclusions is used [1][7][16]. Let us consider a colored target object (a ball), the objective of the fuzzy system is to recognize this color according to its $\{H, S, V\}$ components. The latter are extracted from the image captured by the NAO camera. In this paper, Hough transform is used to detect the ball in the captured image and the average $\{H, S, V\}$ values of the ball's pixels are calculated to be used as the inputs of the fuzzy system.

The finality of the proposed fuzzy system is to exhibit the input-output behavior between the triplet $\{H, S, V\}$ and the recognized color. As illustrated in Fig. 1 the inputs $\{H, S, V\}$ values are processed through a Sugeno fuzzy inference in order to provide an output which is a numerical value indicating the detected color.

In this paper, due to the difficulty of obtaining an exact input-output mathematical model between the triplet $\{H, S, V\}$ and the color and as explained previously, the fuzzy system is based on human perception and observations. Indeed, a human expert distinguishes several zones by observing the color recognition input-output behavior. According to the human expert's observations and analysis, a partitioning of the universes of discourse in fuzzy subsets is given.

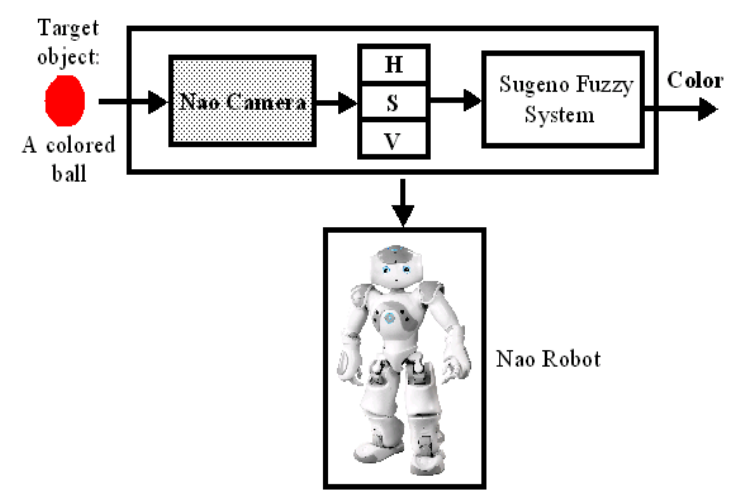

Fig. 1: Sugeno fuzzy system for color recognition.

This partitioning consists of assigning labels allowing the description of the numerical values using words. For example, the human expert interprets the situations by linguistic terms according to whether the saturation of the ball's color is "Normal", "Pale" or "Clear" with regard to the ball color. This classification is easily transcribed into linguistic terms. In this framework, the partitioning of different universes of discourse is illustrated in Fig. [2-4].

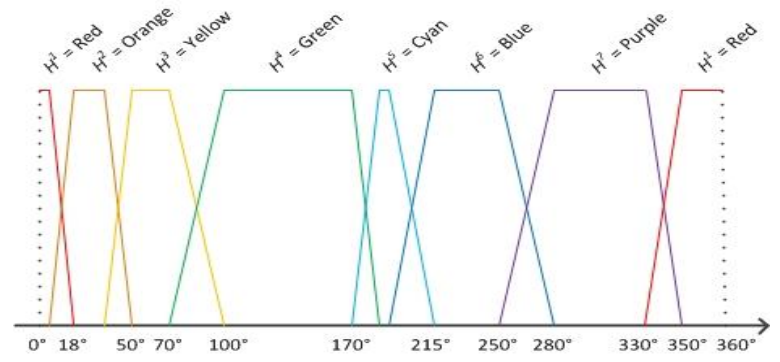

Fig. 2: Membership functions for Hue $(H)$.

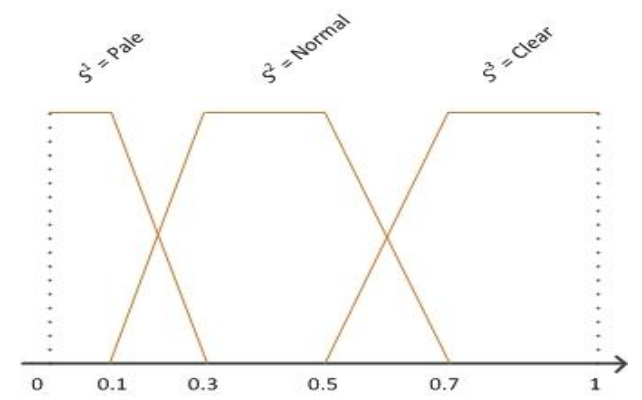

Figure 3: Membership functions for Saturation $(S)$

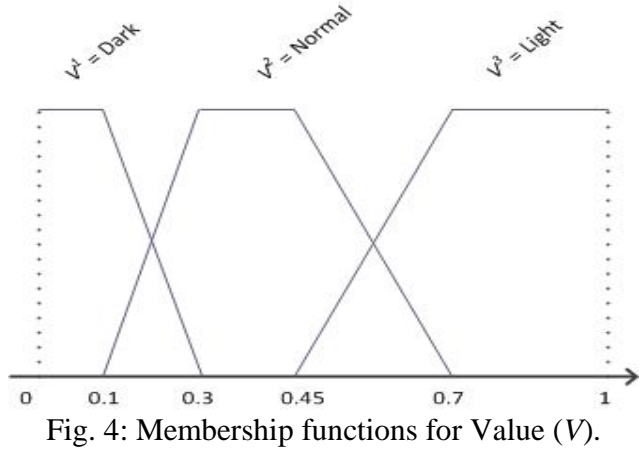

Lastly, according to his/her perception, the human expert determines the output color associated with the $\{H, S, V\}$ inputs. According to the universe of discourse partitioning, the fuzzy system can be formalized as a 
Takagi-Sugeno fuzzy system [1][7][16] given by a collection of rules in the following form:

$$
\begin{gathered}
R^{\left(i_{1}, i_{2}, i_{3}\right)} \quad: \quad \text { If } H \text { is } H^{i_{1}} \text { and } S \text { is } S^{i_{2}} \text { and } V \text { is } V^{i_{3}} \\
\text { then } C=C_{i}
\end{gathered}
$$

where $C_{i}$ is a real value involved in the rule conclusion indexed by $\left(i_{1}, i_{2}, i_{3}\right)$. Indeed, each constant in the conclusion rules represents a color number $C_{i}$ in the set of output colors. In this work, nine colors (see Table 1) are chosen for representing the fuzzy system output, i.e., $\left\{C_{i}\right\}_{i=1, \ldots, 9}=\left\{C_{1}, C_{2}, \ldots, C_{9}\right\}=\{$ Blue, Purple, Pink, Red, Brown, Orange, Yellow, Green, Cyan\}.

\begin{tabular}{|c|c|}
\hline Color $C_{i}$ & $\begin{array}{c}\text { Color } \\
\text { number }\end{array}$ \\
\hline Blue & 1 \\
Purple & 2 \\
Pink & 3 \\
Red & 4 \\
Brown & 5 \\
Orange & 6 \\
Yellow & 7 \\
Green & 8 \\
Cyan & 9 \\
\hline
\end{tabular}

Table 1: Color number.

$H^{i_{1}}, S^{i_{2}}$ and $V^{i_{3}}$ correspond respectively to the $i_{k}$ th

\begin{tabular}{|c|c|c|c|c|}
\hline Rule & $\mathrm{H}$ & $\mathrm{S}$ & $\mathrm{V}$ & $\begin{array}{l}\text { Output } \\
\mathrm{C}_{\mathrm{i}}\end{array}$ \\
\hline 1 & Blue & ANY & ANY & 1 \\
\hline 2 & Green & ANY & ANY & 8 \\
\hline 3 & Cyan & Normal & Normal & 8 \\
\hline 4 & Cyan & Clear & ANY & 9 \\
\hline 5 & Cyan & Pale & ANY & 9 \\
\hline 6 & Cyan & Normal & NOT Normal & 9 \\
\hline 7 & Orange & Clear & Normal & 5 \\
\hline 8 & Orange & Pale & ANY & 6 \\
\hline 9 & Orange & Normal & ANY & 6 \\
\hline 10 & Orange & Clear & NOT Normal & 6 \\
\hline 11 & Yellow & Pale & ANY & 7 \\
\hline 12 & Yellow & Normal & Normal & 8 \\
\hline 13 & Yellow & Normal & NOT Normal & 7 \\
\hline 14 & Yellow & Clear & Normal & 8 \\
\hline 15 & Yellow & Clear & NOT Normal & 7 \\
\hline 16 & Red & Pale & Light & 3 \\
\hline 17 & Red & Pale & NOT Light & 4 \\
\hline 18 & Red & Normal & Dark & 4 \\
\hline 19 & Red & Normal & Normal & 5 \\
\hline 20 & Red & Normal & Light & 3 \\
\hline 21 & Red & Clear & Normal & 5 \\
\hline 22 & Red & Clear & NOT Normal & 4 \\
\hline 23 & Purple & Pale & Light & 3 \\
\hline 24 & Purple & Pale & NOT Light & 2 \\
\hline 25 & Purple & Normal & Light & 3 \\
\hline 26 & Purple & Normal & NOT Light & 2 \\
\hline 27 & Purple & Clear & ANY & 2 \\
\hline
\end{tabular}
linguistic term of the input variables $H, S$ and $V$.

Table 2: The rule base for the Sugeno fuzzy system.
For the input variables and when assuming a strict partitioning of the different universe of discourse illustrated in Fig. [2-4], the output generated by the fuzzy controller is given by:

$$
C=\sum_{\left(i_{1}, i_{2}, i_{3}\right) \in I} \xi^{\left(i_{1}, i_{2}, i_{3}\right)}(H, S, V) \cdot C_{i}
$$

where $\xi^{\left(i_{1}, i_{2}, i_{3}\right)}$ represents the truth value of the premises of the rules and $I=I_{1} \times I_{2} \times I_{3}$ indicates the set of labels representing the rules base, with:

- $i_{1} \in I_{1}=\{1, \ldots, 7\}$,

- $i_{2} \in I_{2}=\{1,2,3\}$

- and $i_{3} \in I_{3}=\{1,2,3\}$.

Thus, the complete base of rules for a fuzzy controller of a transformation module is composed of 63 rules. An example of output values of the rule conclusions obtained from the expertise are summarized in Table 2. In this table ANY means that the corresponding input variable can be substituted by any linguistic value of its set of terms, while Not $X$ means all the terms except $X$.

\subsection{Remarks and practical considerations}

Some remarks and design considerations can now be expressed concerning the proposed methodology presented above:

$\boldsymbol{A}$. In the real-time implementation of recognition using Sugeno fuzzy a threshold $\varepsilon$ is needed. This value is used to indicate whether or not a Sugeno output definitely specifies a color. For example with $\varepsilon$ is 0.2 , an output 4.15 says that the detected color is red (because according to table 1 , the constant number for the color red is 4 , and we have $4-\varepsilon<4.15<4+\varepsilon$ ). So:

If $C \in\left[C_{i}-\varepsilon, C_{i}+\varepsilon\right]$ then $C$ mentions color $C_{i}$. If $C \in\left(C_{i-1}+\varepsilon, C_{i}-\varepsilon\right), \mathrm{C}$ hesitates between $C_{i-1}$ and $C_{i}$ If $C \in\left(C_{i}+\varepsilon, C_{i+1}-\varepsilon\right), \mathrm{C}$ hesitates between $C_{i}$ and $C_{i+1}$ (3)

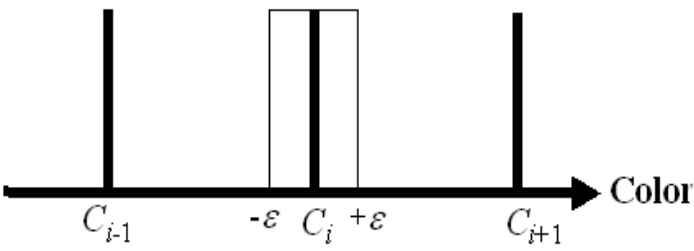

Fig.5: Certainty range of color $C_{i}$.

Obviously and as illustrated in Table 3 where 100 experiments were carried out to detect a red ball, the threshold value influences the color detection rate. Indeed, a small threshold value deteriorates the detection rate and increases the conflict situations (hesitation) between colors. In this case, the reliability and precision of detection are better but the uncertainty on the decision is significant. In other words, the degree of certainty so that a value is included in the interval $\left[C_{i}-\varepsilon, C_{i}+\varepsilon\right]$ is weak. On the other hand, a larger threshold improves the detection rate strategy and weakens the cases of conflict (uncertainty). However, the inaccuracy and the imprecision of the color detection are increased when 
the certainty in decision is significant. In real time applications, a compromise between precision and uncertainty must be taken into account for selecting the threshold value.

\begin{tabular}{|c|c|c|}
\hline $\begin{array}{c}\text { Threshold } \\
\varepsilon\end{array}$ & $\begin{array}{c}\text { Detection } \\
\text { rate }\end{array}$ & $\begin{array}{c}\text { Conflict and } \\
\text { other situations }\end{array}$ \\
\hline 0.05 & $59 \%$ & $41 \%$ \\
0.1 & $61 \%$ & $39 \%$ \\
0.2 & $65 \%$ & $35 \%$ \\
0.3 & $74 \%$ & $26 \%$ \\
0.4 & $80 \%$ & $20 \%$ \\
\hline
\end{tabular}

Table 3: Detection rate for the color red

$\boldsymbol{B}$. It is important to note here that the first and last colors (in our approach they are the Blue (1) and Cyan (9), respectively) are special cases. In order to solve the problem of the first and last color we consider that the colors are distributed in a circular manner so that the first and the last colors are adjacent (this is the case for the Hue in HSV space). Indeed, as illustrated in Fig. 6 this circular representation ensures an adjacency relation between these two colors.

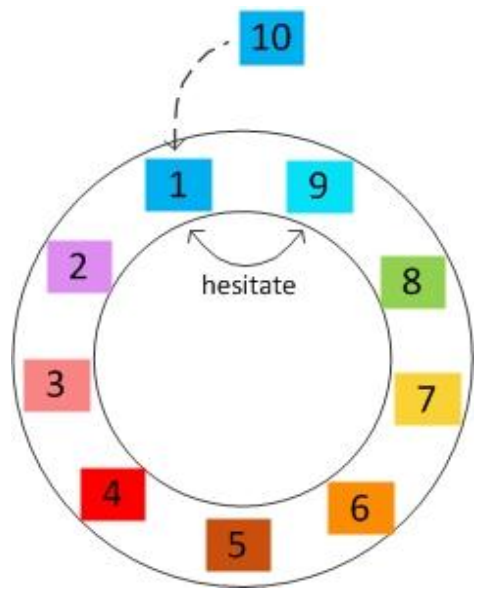

Fig. 6: The first and the last color problem illustration.

In this situation, we cannot process like the other colors. Indeed, the Sugeno system used in this work will provide a numerical output which takes into account the weights of all possible colors. So, if the inputs of the Sugeno fuzzy system activate only the rules containing the colors Blue and Cyan (the first and last one), the output may not be between them but rather some values from 1 to 9 . This situation is not appropriate in terms of human perception of colors. In order to solve this problem, we propose to add a virtual number as illustrated in Fig. 6. In this framework, if there is a hesitation between Blue and Cyan, which have respectively the color number 1 and 9, we use the number 10 for Blue instead of 1 to put the output of the Sugeno formula between these two colors.

$\boldsymbol{C}$. In the proposed methodology, the presence of uncertainties is due to:

- The camera itself and the techniques used to extract data from it (for example the use of Hough transform to detect a ball in a captured image).
- The chosen threshold value in the recognition mechanism,

- The change in operating conditions for image acquisition (lighting, orientation of the object, orientation of the robot ... etc.),

- The reliability of the camera according to the detected color.

In this framework, the results given by the fuzzy system can be conflictual. Indeed, as illustrated in Fig. 7 the fuzzy system gives the following results in three different operating conditions for a red ball:

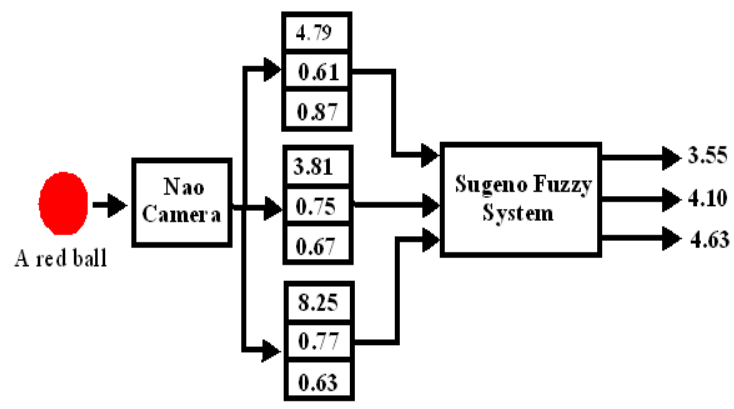

Fig.7: Color detection in different operating conditions.

In this situation and as illustrated in Fig. 8, the second case (color $=4.10)$ can be interpreted as a red color. However, the first and third cases present respectively conflictual situations between red and pink and between red and brown. In this case, a decision cannot be made.

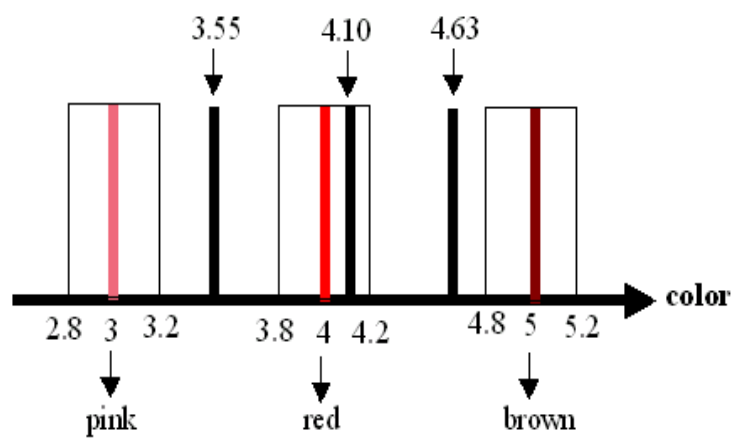

Fig.8: Color detection according to the threshold value

More generally, in this paper the imprecision of the recognition system is assumed to be reasonable and equal to \pm 1 color. In other words, the detected color is always in the interval $\left[C_{i-1}, C_{i+1}\right]$. The values that are outside of this interval are not considered and penalize the performance of the system of recognition.

In this framework and as illustrated in Fig. 9, for a given triplet $\{H, S, V\}$ related to a color $C_{i}$ (when the robot is asked to find the color $C_{i}$ ), the fuzzy system can give one situation from the set of possibilities: $\left\{C_{i}, C_{i-1}\right.$, $C_{i+1}$, conflict between $C_{i}$ and $C_{i-1}$, conflict between $C_{i}$ and $\left.C_{i+1}\right\}$. 


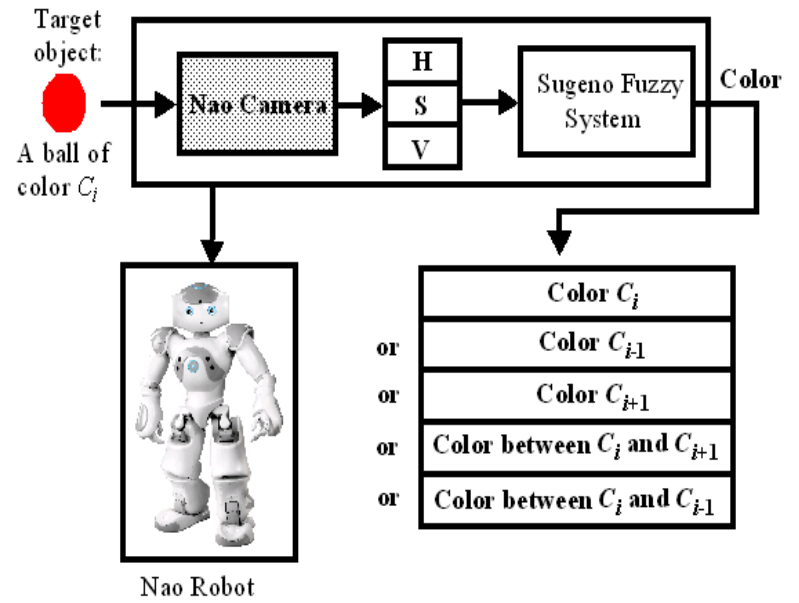

Fig. 9: Possible outputs for the fuzzy system

In order to overcome the conflict situations and improve the detection strategy, we propose to add more displaced cameras. These cameras also use Sugeno fuzzy system to recognize the same target object. For the sake of simplicity and without loss of generality, only one second camera is used in this paper. In this situation and in presence of conflicts between the two cameras, evidence theory is used to fuse information in order to have a better color recognition decision. The concept is detailed in the next section.

\section{Evidence Theory For Multiple Cameras Fusion}

\subsection{Fusion context}

The overall process of the fusion of two cameras is described in the Fig. 10. Initially, the NAO robot is asked to find an object with the color $C_{i}$. Both the NAO camera and the displaced IP camera will use the Sugeno fuzzy system to identify the color of the detected object. The IP camera communicates with the robot through a WIFI network. In presence of conflicts between the cameras, their Sugeno outputs will be used as the inputs for the decision system which implements Evidence theory to give the final color decision. In this work, the fuzzy system and the decision mechanism are implemented in the NAO robot.

It is important to note here that the NAO robot has two cameras, but they have different viewing angles, so they cannot capture the same object at the same time. That is the reason why in this work we chose another IP camera as the second information source.

According to the fuzzy system detailed in the previous section, for a given color $C_{i}$, the following space of discernment is used:

$$
\Omega_{i}=\left\{C_{i-1}, C_{i}, C_{i+1}\right\}
$$

Consequently, for a given color $C_{i}$ we can construct the power set, which takes into account all the possible hypotheses:

$$
\begin{aligned}
P_{i}=2^{\Omega_{i}}= & \left\{\{\varnothing\},\left\{C_{i-1}\right\},\left\{C_{i}\right\},\left\{C_{i-1}, C_{i}\right\},\left\{C_{i+1}\right\},\right. \\
& \left.\left\{C_{i-1}, C_{i+1}\right\},\left\{C_{i+1}, C_{i}\right\}, \Omega_{i}\right\}
\end{aligned}
$$

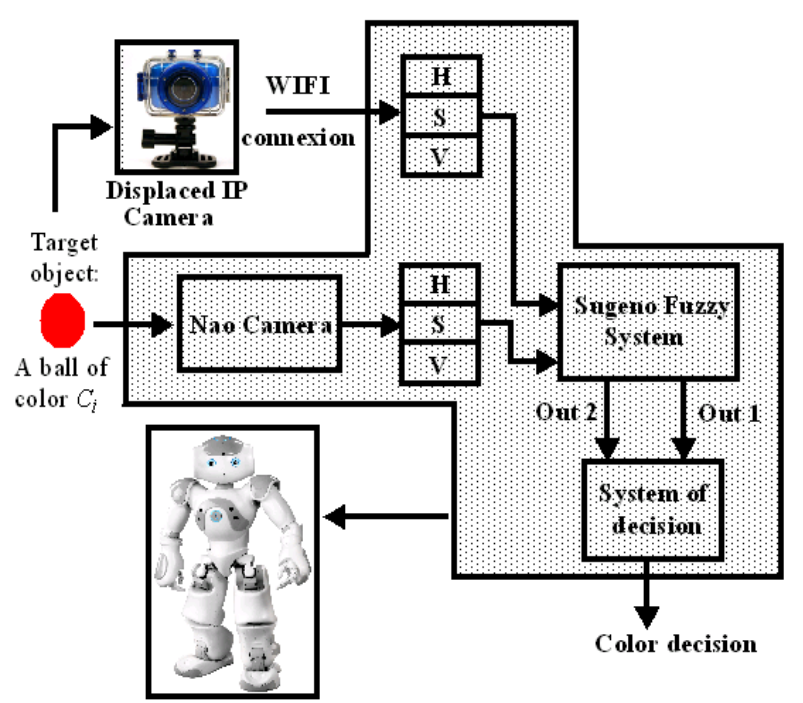

Fig. 10: Fusion architecture mechanism of two cameras.

As illustrated in Fig. 11, for a given color $C_{i}$ the decision system is composed of three stages. Firstly, the mass vectors for each Sugeno system output related to each camera are constructed. After that, a combination operator is used to combine and fuse those masses and finally a decision operator is used to give the final output color.

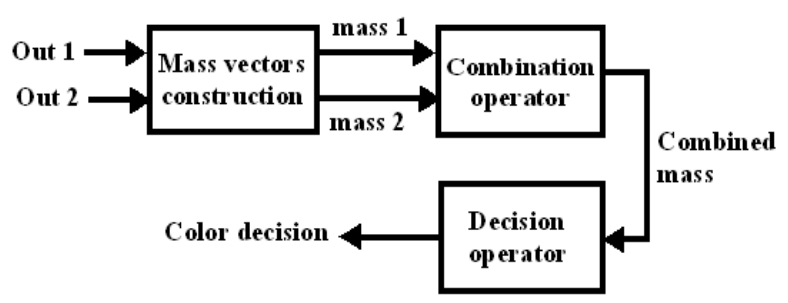

Fig. 11: Decision strategy principle

\subsection{Construction of Fuzzification-based masses}

The first step in the proposed decision methodology consists of determining appropriate mass functions which allow describing the belief from information sources (cameras). In other words, we have to build a function related to the color $\mathrm{Ci}$ for each camera:

$$
\begin{aligned}
& m_{\text {camera }}^{i}: 2^{\Omega_{i}} \rightarrow[0,1], \text { such as: } \\
& \sum_{X \in 2^{\Omega_{i}}} m_{\text {camera }}^{i}(X)=1, \text { and }: m_{\text {camera }}^{i}(\varnothing)=0
\end{aligned}
$$

In fact, from a given Sugeno output (a numerical number), we will infer the belief for each hypothesis in (5). To do this, a Fuzzy-based approach is proposed as in Fig. 12. The mass values of $\left\{C_{i}\right\},\left\{C_{i-1}\right\}$ and $\left\{C_{i+1}\right\}$ are constructed with trapezoidal membership functions based on the value of the threshold mentioned in section 2.2. The triangular membership functions are used for $\left\{C_{i}, C_{i-1}\right\}$ and $\left\{C_{i}, C_{i+1}\right\}$. The given Sugeno output is fuzzified and the degree of membership with each hypothesis describes its mass value. Obviously, other 
forms (more interesting forms) of membership functions can be used.

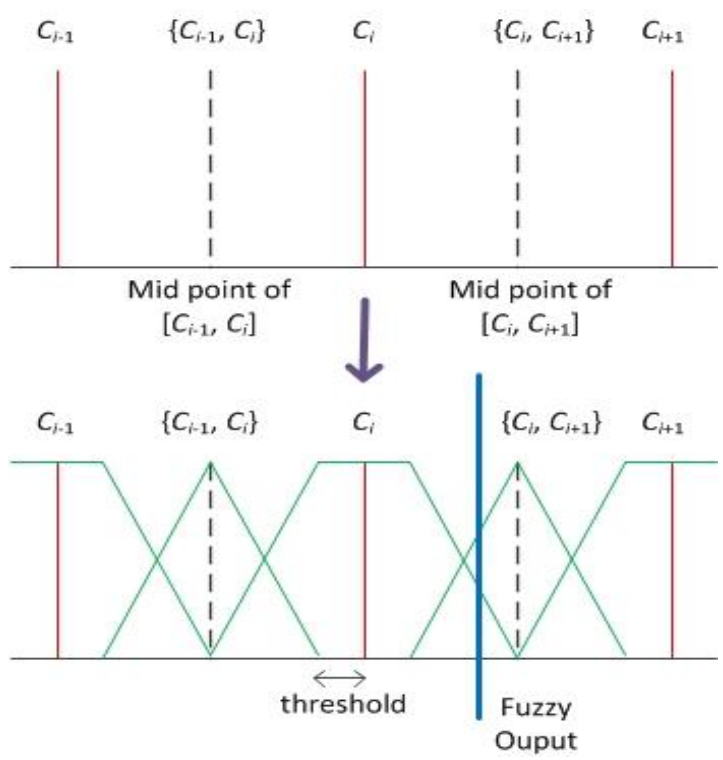

Fig. 12: Fuzzification based masses construction.

It is important to note here that due to the natural order of the colors, in absolute terms we have:

$$
\begin{aligned}
& m_{\text {camera }}^{i}\left(\left\{C_{i-1}, C_{i+1}\right\}\right)=0 \\
& m_{\text {camera }}^{i}\left(\left\{\Omega_{i}\right\}\right)=0
\end{aligned}
$$

\subsection{Combination operator}

The fuse of mass functions resulting from various sources (cameras in our work) can be realized according to several combination operators. Initially, the first rule suggested by Dempster and recovered by Shafer is the normalized conjunctive rule [13]. The normalization in the DS rule masks the total conflict and is thus interesting only in a closed world for the combination of relatively reliable sources. In the literature, several combination operators have been proposed. For instance, Florea [6] proposes a rule based on a weighted sum between the conjunctive and disjunctive rules according to the total conflict. In his rule, Yager [18] distributes the total conflict on total ignorance (i.e on the mass of $\Omega$ ) in order to remain in a closed world and considers that we do not know anything in conflict situations. Dubois and Prade [5] propose a powerful rule in the case where information of the sources is stated on singletons. Indeed, this method proposes a finer management of the conflict by distributing the partial conflict on partial ignorance. This method is more adapted in presence of unreliable sources.

In this paper, as the sources are relatively reliable and according to our experience results where all the operators quoted previously were tested, it seems that the DS operator represents the best compromise. So, for each color $C_{i}$ and for each camera the following DS operator is used:

$$
\forall X \in 2^{\Omega_{i}}:\left\{\begin{array}{l}
m_{D S}^{i}(X)=\frac{1}{1-k} \sum_{Y_{1} \cap \ldots, \cap Y_{s}=X} \Pi_{j=1}^{s} m_{j}^{i}\left(Y_{j}\right), X \neq \varnothing \\
0, X=\varnothing
\end{array}\right.
$$

where $k$ represents the conflict between sources and $Y_{j}$ is the information given by the source $j$.

\subsection{Decision Operator}

In order to preserve a maximum of information, it is preferable to remain at a creed level (manipulating functions of belief) during the combination stage of combination in order to make the decision on functions of belief resulting from the combination. Generally, if the decision taken by the maximum of belief is too pessimistic, the decision resulting from the maximum of plausibility is very often too optimistic, the maximum of the pignistic probability, introduced by Smets [15], remains the most used compromise. So, in this paper the maximum of the pignistic probability is used as a decision operator.

Therefore, with each color $C_{i}$ and for each camera the maximum of the pignistic probability given by following equation is used.

$$
\operatorname{Bet}_{m^{i}}(X)=\frac{1}{1-m(\varnothing)} \sum_{X \in A} \frac{m^{i}(A)}{|A|}
$$

where $|A|$ represents the cardinality of a subset $A \subseteq \Omega_{i}$.

\subsection{Masses Improvement by Reliabilities}

Due to the fact that each camera has its own reliability and they may not be the same for each color, we propose to improve the sensitivity of the decision system by considering the discounting factors applied for each camera with each considered color.

Let $\alpha_{i}$ be the discounting factor for the color $C_{i}$ with a camera. This parameter is considered as the detection rate for the target color $C_{i}$. The discounting factors are applied to reduce the belief in the mass values from each information source as follows:

$$
\left\{\begin{array}{l}
m_{\alpha_{i}}^{i}(X)=\alpha_{i} \cdot m^{i}(X) \\
m_{\alpha_{i}}^{i}\left(\Omega_{i}\right)=1-\alpha_{i} \cdot\left(1-m^{i}\left(\Omega_{i}\right)\right)
\end{array}\right.
$$

where $m_{\alpha_{i}}^{i}(X)$ is the mass value of the color for the hypothesis $X$ after being discounted by the factor $\alpha_{i}$.

In our application an image data base composed of 100 images for each color and for each camera (100x9 images / camera) under different changes of lighting conditions is used for determining the discounting factors for each camera (NAO and IP).

\subsection{Illustrative Example}

For instance, the NAO robot is asked to find a required red ball. According to table 1, the constant number for the color red is 4 . During the detection process, the two cameras (NAO and IP) capture the same object but give different Fuzzy Sugeno results, i.e.,

$$
\left\{\begin{array}{l}
\text { Sugeno }_{\mathrm{NAO}}=4.63 \\
\text { Sugeno }_{\mathrm{IP}}=3.60
\end{array}\right.
$$


The conflict $k$ between the two cameras is equal to 0.382 . The reliability of the NAO camera and the IP camera for the color red are respectively 0.73 and 0.51 . In this example, the threshold $\varepsilon$ is chosen to be equal to 0.2 .

According to the values of the Sugeno system outputs, the robot and the IP camera hesitate respectively between \{Red (4), Brown (5)) and between \{Pink (3), $\operatorname{Red}(4)$ ). Based on the method of mass construction discussed in the previous section, we are able to build the mass vectors for those information sources. Table 4 shows:

- The mass vectors results without camera reliability $m_{\mathrm{NAO}}$ and $m_{\mathrm{IP}}$.

- The mass vectors results with camera reliability $\left(m_{\mathrm{NAO}}\right)_{\mathrm{R}}$ and $\left(m_{\mathrm{IP}}\right)_{\mathrm{R}}$

- The combined mass vector using the DempsterShafer operator $m_{D S}$.

- The decision given by the maximum of pignistic probability $P P$.

\begin{tabular}{|c|c|c|c|c|c|c|}
\hline & $m_{\mathrm{NAO}}$ & $m_{\mathrm{IP}}$ & $\left(m_{\mathrm{NAO}}\right)_{\mathrm{R}}$ & $\left(m_{\mathrm{IP}}\right)_{\mathrm{R}}$ & $m_{\mathrm{DS}}$ & $P P$ \\
\hline$\{\varnothing\}$ & 0.00 & 0.00 & 0.00 & 0.00 & 0.00 & \\
$\{3\}$ & 0.00 & 0.00 & 0.00 & 0.00 & 0.00 & 0.11 \\
$\{\mathbf{4}\}$ & $\mathbf{0 . 0 0}$ & $\mathbf{0 . 3 3}$ & $\mathbf{0 . 0 0}$ & $\mathbf{0 . 1 7}$ & $\mathbf{0 . 3 1}$ & $\mathbf{0 . 5 3}$ \\
$\{3,4\}$ & 0.00 & 0.67 & 0.00 & 0.34 & 0.11 & \\
$\{5\}$ & 0.44 & 0.00 & 0.32 & 0.00 & 0.18 & 0.36 \\
$\{3,5\}$ & 0.00 & 0.00 & 0.00 & 0.00 & 0.00 & \\
$\{4,5\}$ & 0.56 & 0.00 & 0.41 & 0.00 & 0.24 & \\
$\{3,4,5\}$ & 0.00 & 0.00 & 0.27 & 0.49 & 0.16 & \\
\hline Decision & & & & & $\mathbf{4}$ \\
& & & & & & Red \\
\hline
\end{tabular}

Table 4: Example of mass construction and fusion.

In table 4 we can see that the decision after the fusion is color 4 (the red color). So the robot can make a decision according to the DS theory and recognizes the target object.

\section{Application and Experimentation}

\subsection{Overview of the application}

The implemented application is illustrated in Fig. 10. Firstly, the NAO robot, through a communication channel with humans (here we give it oral commands), is demanded to search the required colored objects (colored balls). Thereafter, it walks around while using one of its cameras on its head to capture surrounding images. At the same moment, the IP camera which is in communication with the robot (WIFI network) scans in the same view. The robot and NAO communicate through a local wireless connection and both use the Sugeno fuzzy system to determine the color of the same detected balls.

Whenever the results from the two cameras are conflictual we apply the Evidence Theory to make a decision on the output color. Finally, the NAO robot will say that it has found the target object with the required color. If there are total conflicts between the two cameras and we cannot make a final decision despite using fusion methods, the robot will say that it is hesitating about the results and needs more information to make decisions. In this case we can consider adding other cameras or giving more priorities on one camera.

\subsection{Experimentation results}

Table 5 shows the results from experiments in which we have make tests with 100 colored balls for each color and for each camera. In Table 5, the first column lists all the colors, the second and the third columns present the quality of recognition for the NAO camera and the IP camera, respectively, using only the Sugeno fuzzy system. The final column indicates the improvement in the recognition quality by using Evidence Theory in fusing information from the two cameras. Note that the last row is the average value of the upper ones. The threshold value used for this experimentation is 0.2 .

\begin{tabular}{|c|c|c|c|}
\hline Color & $\begin{array}{c}\text { Sugeno } \\
\text { Fuzzy } \\
\text { System } \\
\text { (NAO) }\end{array}$ & $\begin{array}{c}\text { Sugeno } \\
\text { Fuzzy } \\
\text { System } \\
\text { (IP) }\end{array}$ & $\begin{array}{c}\text { Fusion of the } \\
\text { cameras with } \\
\text { reliabilities }\end{array}$ \\
\hline Blue & $98 \%$ & $64 \%$ & $100 \%$ \\
Purple & $93 \%$ & $95 \%$ & $100 \%$ \\
Pink & $44 \%$ & $40 \%$ & $69 \%$ \\
Red & $65 \%$ & $52 \%$ & $90 \%$ \\
Brown & $70 \%$ & $51 \%$ & $88 \%$ \\
Orange & $68 \%$ & $76 \%$ & $96 \%$ \\
Yellow & $37 \%$ & $44 \%$ & $87 \%$ \\
Green & $62 \%$ & $62 \%$ & $77 \%$ \\
Cyan & $38 \%$ & $26 \%$ & $98 \%$ \\
\hline Avg. & $\mathbf{6 3 . 8 9 \%}$ & $\mathbf{5 6 . 6 7 \%}$ & $\mathbf{8 9 . 4 4 \%}$ \\
\hline
\end{tabular}

Table 5: color recognition performances.

According to these results it can be stated that the color recognition performance has been increased using the DS theory. Indeed, the rate of recognition has been improved by $26 \%$ for the NAO camera and $33 \%$ for the IP one. The situations of non-color recognition ( 10\%) are essentially due to the presence of total conflict between sources and/or the existence of some fuzzy system outputs outside the space of discernment In this case, as the DS combination operator is only powerful in a closed world and using relatively reliable sources, other operators dealing with these last problems will be considered in the future (for example the Yager and Dubois-Prade operators). Indeed, cohabitation between combination operators can be an interesting tool to overcome these problems. Moreover, other techniques of mass vectors construction (like the use of a learning data base) can be used.

\section{Conclusion}

In this paper a methodology of colored object recognition for the NAO humanoid robot is proposed. The designed strategy is based on the collaboration between a Sugeno fuzzy system and a decision mechanism based on the DS theory. Indeed, to improve the quality of recognition for the fuzzy system, we propose to use 
multiple cameras (two cameras in our work) to help the robot make a decision. In presence of conflictual situations between the cameras, the DS theory is used to fuse the information so that better decisions are made.

Experiment results show the validity of our approach and demonstrate that the fusion of multiple cameras dramatically improves the quality of recognition in comparison with using only the fuzzy system for each camera separately.

Future work will focus on the extension of this approach to 3D colored object recognition using a Kinect camera where fuzzy and gradual interval arithmetic will be used [2][3].

\section{References}

[1] R. Boukezzoula, S. Galichet S. and L. Foulloy, Nonlinear internal model control: application of inverse model based fuzzy control. Fuzzy Systems, IEEE Transactions on Vol. 11, N6, pp. 814-829, 2003.

[2] R. Boukezzoula, S. Galichet, and A. Bisserier, A Midpoint-Radius approach to regression with interval data. International Journal of Approximate Reasoning Vol. 52, № 9, pp. 1257-1271, 2011.

[3] R. Boukezzoula, S. Galichet, L. Foulloy and M. Elmasry, Extended gradual interval (EGI) arithmetic and its application to gradual weighted averages. Fuzzy Sets and Systems Vol. 257, pp. 67-84, 2014.

[4] A.P. Dempster, Upper and lower probabilities induced by a multivalued mapping. The annals of mathematical statistics, pp. 325-339, 1967.

[5] D. Dubois and H. Prade, Representation and combination of uncertainty with belief functions and possibility measures. Computational Intelligence Vol. 4, N 3, pp. 244-264, 1988.

[6] M.C. Florea, Combinaison d'informations hétérogènes dans le cadre unificateur des ensembles aléatoires: approximations et robustesse. Thèse de doctorat. Université Laval, 2007.

[7] S. Galichet, R. Boukezzoula and L. Foulloy, Explicit analytical formulation and exact inversion of decomposable fuzzy systems with singleton consequents. Fuzzy Sets and Systems, Vol. 146, N 3 pp. 421-436, 2004.

[8] X. Han, et al., An approach of color object searching for vision system of soccer robot. Robotics and Biomimetics, IEEE ROBIO, 2004.

[9] O. Kucuktunc and Z. Daniya, Fuzzy color histogram-based CBIR system. Proceedings of 1st International Fuzzy Systems Symposium, 2009.

[10] S.G. Lee, K. Kwang-Baek and C. Eui-Young, Color Inference Using an Enhanced Fuzzy Method. Red (R), Vol. 330, 2013.

[11] Z. May and M. H. Amaran, Automated oil palm fruit grading system using artificial intelligence. Int. J. Eng. Sci, Vol. 11, pp. 30-35, 2011.

[12] V. Rahmani, V. Rostami, Adaptive Color Mapping for NAO Robot Using Neural Network. ACSIJ Advances in Computer Science: an International Journal, Vol. 3, N $\mathrm{N}^{\circ}$ 5-11, 2014.
[13] G. Shafer, A mathematical theory of evidence., Vol. 1. Princeton: Princeton university press, 1976.

[14] L. Shamir, Human Perception-based Color Segmentation Using Fuzzy Logic. IPCV 2, pp. 96-502, 2006.

[15] Ph. Smets, Constructing the Pignistic Probability Function in a Context of Uncertainty. UAI. Vol. 89. 1989.

[16] T. Takagi and Sugeno M., Fuzzy identification of systems and its applications to modeling and control. IEEE Transactions on Systems, Man and Cybernetics, Vol. 1, pp. 116-132, 1985.

[17] T. Peter J.S. Russel and P. J. Wolfs. Robustness of colour detection for robot soccer. ICARCV, 2002.

[18] R.R. Yager, On the Dempster-Shafer framework and new combination rules. Information sciences, Vol. 41, N², pp. 93-137, 1987.

[19] C. Wong, A real-time color recognition technique. Color Research and Application, 1995. 